\title{
A local average distance descriptor for flexible protein structure comparison
}

\author{
Hsin-Wei Wang ${ }^{1 \dagger}$, Chia-Han Chu ${ }^{2 \dagger}$, Wen-Ching Wang ${ }^{2,3}$ and Tun-Wen Pai ${ }^{1 *}$
}

\begin{abstract}
Background: Protein structures are flexible and often show conformational changes upon binding to other molecules to exert biological functions. As protein structures correlate with characteristic functions, structure comparison allows classification and prediction of proteins of undefined functions. However, most comparison methods treat proteins as rigid bodies and cannot retrieve similarities of proteins with large conformational changes effectively.

Results: In this paper, we propose a novel descriptor, local average distance (LAD), based on either the geodesic distances (GDs) or Euclidean distances (EDs) for pairwise flexible protein structure comparison. The proposed method was compared with 7 structural alignment methods and 7 shape descriptors on two datasets comprising hinge bending motions from the MolMovDB, and the results have shown that our method outperformed all other methods regarding retrieving similar structures in terms of precision-recall curve, retrieval success rate, R-precision, mean average precision and $\mathrm{F}_{1}$-measure.

Conclusions: Both ED- and GD-based LAD descriptors are effective to search deformed structures and overcome the problems of self-connection caused by a large bending motion. We have also demonstrated that the ED-based $L A D$ is more robust than the GD-based descriptor. The proposed algorithm provides an alternative approach for blasting structure database, discovering previously unknown conformational relationships, and reorganizing protein structure classification.
\end{abstract}

\section{Background}

Protein structure comparison plays an important role in predicting functions of novel proteins [1] and several methods have been developed for pairwise [2-8] and multiple [9-16] comparisons. Most existing methods of structure comparison treat proteins as rigid bodies; however, protein structures are flexible and conformationally changeable in response to binding another molecules relating with biological functions such as immune protection, enzymatic catalysis and cellular locomotion $[17,18]$. Such structural variations caused rigid-body algorithms unable to generate correct alignments or retrieve similar structures with large deformations. Therefore, flexibility of proteins should be taken into account when comparing structures and searching for similarities to a query structure.

\footnotetext{
* Correspondence: twp@mail.ntou.edu.tw

${ }^{\dagger}$ Equal contributors

'Department of Computer Science and Engineering, National Taiwan Ocean University, Keelung, Taiwan

Full list of author information is available at the end of the article
}

\section{Alignment methods}

Flexible structure comparison has received much attention in recent years. For instance, FlexProt found congruent rigid fragment pairs between two proteins and the flexible regions (hinges), and then a clustering procedure was performed to join consecutive fragment pairs into congruent domain pairs $[19,20]$. FATCAT connected aligned fragment pairs based on a dynamic programming algorithm which introduced penalty scores for gaps and twists between consecutive aligned fragment pairs [21]. Compared with FlexProt, FATCAT generates alignments with less twists but similar root mean square deviations (RMSDs) and lengths. The TOPS++FATCAT algorithm reduced the number of aligned fragment pairs during FATCAT comparison processes by applying topological constraints obtained from the alignment of secondary structure elements (SSEs) of TOPS + [22]. Therefore, TOPS++FATCAT is more than 10 times faster compared to FATCAT. Both FlexProt and FATCAT are sequential alignment algorithms thus unable to identify non-sequential alignments. FASE [23] and 
FlexSnap [24] were designed to tackle the problem of nonsequential flexible structure alignment. FASE compares structures starting from aligned pairs of SSEs with an assumption that an optimal superposition of pairs of structures must have at least one pair of well-aligned SSEs. FlexSnap applies a greedy algorithm for connecting aligned fragment pairs and possesses competitive results against other state-of-the-art pairwise comparison methods. Matt, one of the most popular and accurate flexible multiple structure alignment methods, is also based on the approach of chaining aligned fragment pairs which are allowed translations and rotations during assembling $[25,26]$.

\section{Non-alignment methods}

The alignment/superposition based comparison methods are inefficient for blasting similar structures from a structure database in real-time [27]. Therefore, several nonalignment approaches based on different descriptors of molecular shapes were proposed. These descriptors are usually represented by histograms or vectors, and a similarity score between two molecules is calculated from corresponding descriptors without any alignment [28,29]. For example, Daras et al. applied the spherical trace transform method to produce rotational invariant descriptor vectors constituted by weighted geometry- and attribute-based vectors for protein classification [30]. The 3D Zernike descriptor represented a protein structure by 121 numbers based on a series expansion of 3D functions for fast retrieval of similarities, and which demonstrated that lowresolution structures were also applicable [27,31]. Abu Deeb et al. proposed a global descriptor on protein surface, and which was constructed from local patch descriptors defined by residue-specific distance distributions between $\mathrm{C} \alpha$ atoms and the numbers of pairwise residue co-occurrences within each surface patch [32]. Yin et al. compared local surface of proteins by geometric fingerprints of each surface patch [33]. A fingerprint consists of 60 (4 by 15 ) bins corresponding to the geodesic-distancedependent distribution of curvatures.

Nevertheless, most non-alignment methods treated proteins as rigid bodies and neglected flexibility of protein conformations required for performing biological functions. To confront the issue of flexibility, Liu and Fang et al. proposed several histogram based descriptors for flexible molecules comparison. For instance, a local diameter descriptor for depicting the local characteristics of boundary points [34], and another descriptor, inner distance, defined as the shortest path between landmark points $[28,35]$. Both methods are sensitive to self-connection problems during molecular shape deformation. Accordingly, an improved method named Diffusion Distance Shape Descriptor (DDSD) was proposed, which is based on an average distance instead of the shortest distance between two landmark points [36]. Although DDSD is superior to local diameter, inner distance and other descriptors in terms of retrieving similar protein structures, its performance is still unsatisfied with an $\mathrm{F}_{1}$-measure of $37.04 \%$.

\section{Proposed method}

Non-alignment or descriptor based approaches are generally fast enough to search a large database in a real-time manner, but do not provide corresponding information of residues which might provide crucial information for biologists. Combining the ideas of alignment and descriptor based approaches, we propose a novel and efficient descriptor called local average distance (LAD) which is based on either geodesic distances (GDs) or Euclidean distances (EDs) for pairwise flexible protein structure comparison. Each protein structure is firstly transformed into its corresponding LAD profile, and the similarity between two proteins is calculated according to pairwise local alignment on transformed profiles. The Hinge Atlas and Hinge Atlas Gold datasets [37] from the MolMovDB [38] were employed to evaluate the performance of proposed LAD descriptors and to compare with several non-alignment and rigid/flexible structure alignment methods.

\section{Methods}

The proposed protein structure comparison algorithm is based on the LAD profile which is built from pairwise residue distances (ED or GD) within a protein. The workflow of generating profiles from atomic coordinates of proteins is shown in Figure 1. The similarity between two proteins is determined by a local pairwise alignment of their corresponding LAD profiles. The core procedures can be decomposed into triangular surface construction, surface simplification, ED/GD calculation, profile construction and profile comparison. Details of each step are introduced in the following sections.

\section{Triangular surface construction and simplification}

The solvent-accessible surface (SAS) [39] and solventexcluded surface $[40,41]$ (SES, also known as molecular surface or Connolly surface) are the most widely used definitions for protein surface analysis. Each atom of a protein is represented as a sphere with its van der Waals radius. The SAS is traced out by the center of a solvent probe sphere rolling over the spherical atoms, whereas the SES is formed by the inward-facing surface of the probe consisting of contact surface and re-entrant surface. For a more complete description of both SAS and SES please refer to [42]. Many algorithms have been developed to build SAS and/or SES such as Gauss-Bonnet theorem [43], level-set [44], alpha shape [45,46], beta shape [47], Euclidean distance transform [48], ray-casting [49] et al. [50-52]. One common area-based method defines a residue as a surface residue if its surface area is greater than a specific threshold $[46,53]$. The other area-based methods 

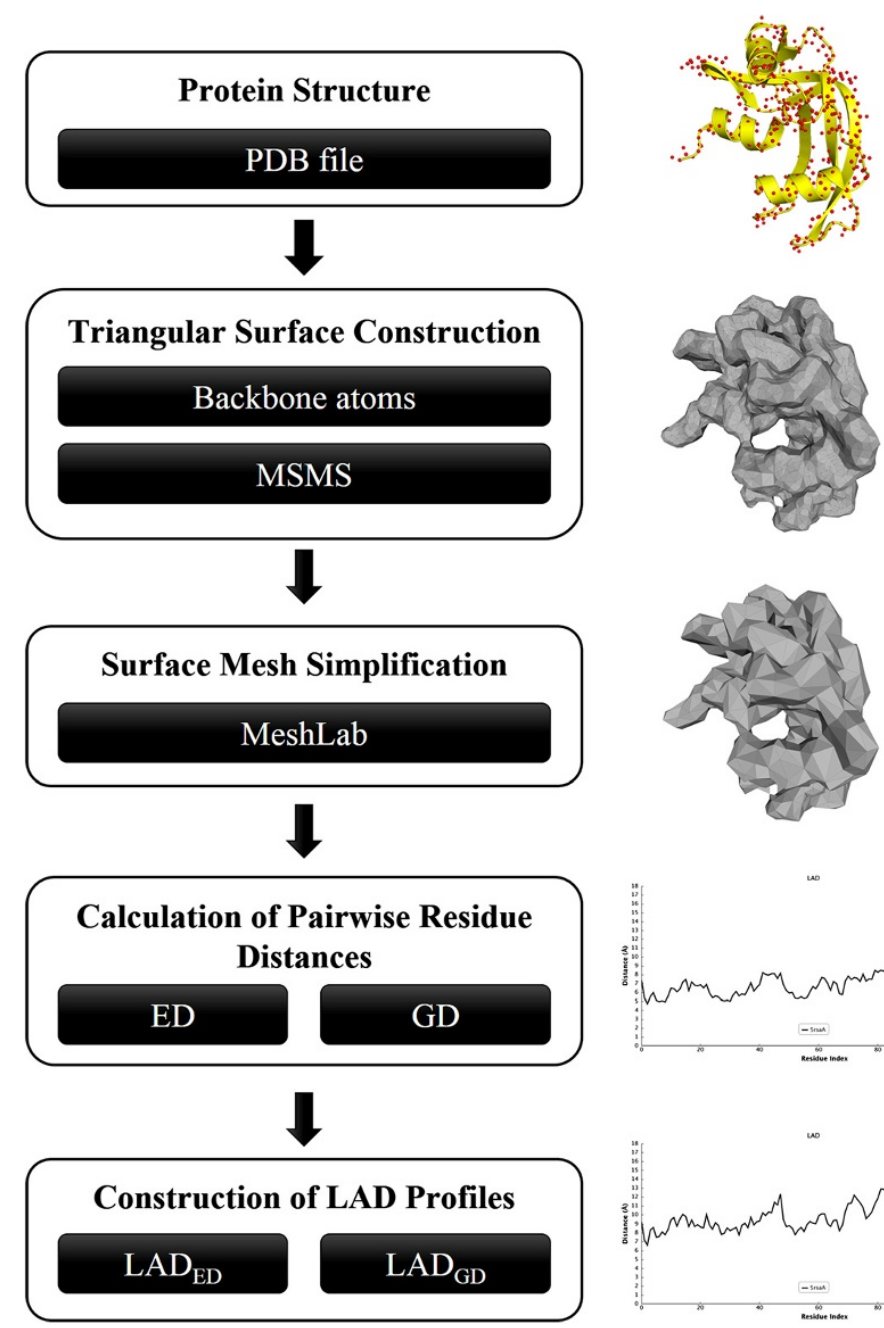

(a)

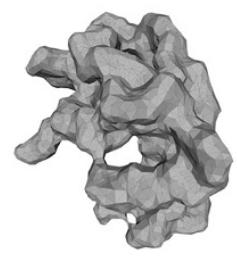

(b)

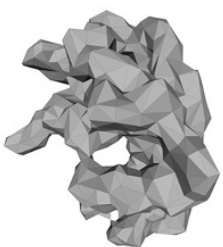

(c)

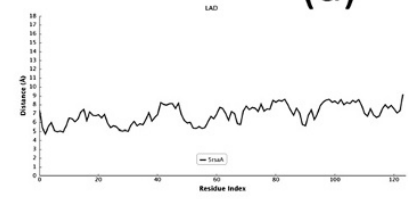

(e)

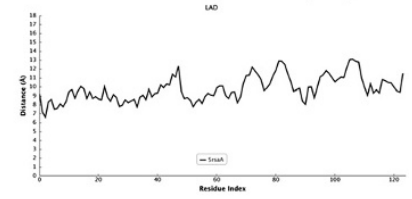

Figure 1 Flowchart of LAD profile generation for the protein 5rsa:A. (a) Yellow ribbons display the secondary structures and red spheres represent the backbone atoms ( $\mathrm{N}, \mathrm{Ca}, \mathrm{C}, \mathrm{O})$ of each residue. (b) The MSMS-generated triangular surface. (c) The simplified surface which is created by MeshLab. (d) and (e) are $L A D$ profiles of the input protein for $E D\left(L A D_{E D}\right)$ and $G D\left(L A D_{G D}\right)$ respectively.

consider a residue with relative solvent accessibility larger than a threshold as a surface residue $[54,55]$. The relative solvent accessibility is defined by taking a residue's solventaccessible area divided by the maximum area of that residue $[56,57]$. In recent years, novel atom-depth-based approaches were proposed as alternative ways to define surface residues [58,59]. Different algorithms employed various definitions of atom depth which could be defined as the distance of an atom from the nearest water molecule surrounding the protein, from the molecular surface, or from its closest solvent-accessible neighbor [60].

The input for building an LAD profile is a standard PDB file. Owing to the requirement of triangular surface meshes for GD calculation, one of the most used and fastest surface program, MSMS v2.6.1 [61], is applied to construct triangular surface meshes from coordinates of all backbone atoms of the protein (Figure 1a). All the parameters of MSMS are remained as default settings. This tool usually generates high resolution meshes (Figure 1b) for proteins. However, it is time-consuming and memory exhausted during the calculation of GDs among mesh vertices. To reduce the resolution of MSMS-generated meshes, an open source tool, MeshLab v1.3.2 (http://meshlab.sourceforge. net/), is adopted to downsample original meshes. The outputs of MSMS are converted into Polygon File Format (Stanford Triangle Format) as MeshLab's inputs. The algorithm of Quadric edgecollapse, a variant of the well-known quadric error metric algorithm [62], is employed to simplify meshes (Figure 1c). As a result, the face number of each MSMS-generated mesh could be reduced by $85 \%$ generally in this research.

\section{Calculation of pairwise residue distances}

The simplified meshes are then used to identify surface residues, and the GDs and EDs of surface residue pairs can be obtained. Each vertex of a simplified mesh 
belongs to the closest backbone atom of the protein. In other words, an atom could possess more than one vertex. We defined that the vertices belong to an atom as the associated vertices of that atom. A residue is regarded as a surface residue if its backbone atoms have at least one vertex.

GD is the shortest path along the surface from source to destination points. We adopted the previously published open source program provided by Danil Kirsanov (http://code.google.com/p/geodesic/) to calculate GDs between any two vertices from simplified meshes. The GD between two atoms, $a_{i}$ and $a_{j}$ is defined by taking average of GDs from all associated vertices and represented as the following:

$$
G D\left(a_{i}, a_{j}\right)=\frac{\sum_{x=1}^{M} \sum_{y=1}^{N} G D\left(v_{i}^{x}, v_{j}^{y}\right)}{M \times N}
$$

where $G D\left(a_{i}, a_{j}\right)$ is the average GD from the $i^{\text {th }}$ atom to the $j^{\text {th }}$ atom, $v_{i}^{x}$ and $v_{j}^{y}$ represent the $x^{\text {th }}$ vertex of the $i^{\text {th }}$ atom and the $y^{\text {th }}$ vertex of the $j^{\text {th }}$ atom respectively. The symbols $M$ and $N$ indicate the number of vertices associated with the $i^{\text {th }}$ atom and the $j^{\text {th }}$ atom, and $G D\left(v_{i}^{x}, v_{j}^{y}\right)$ is the GD from vertex $v_{i}^{x}$ to vertex $v_{j}^{y}$. The atoms possessing no associated vertices won't be considered, hence $M$ and $N$ must be strictly larger than zero. In contrast to the measurement of GD, an ED between two atoms can be easily obtained from their coordinates. Once the two different distance measures between any two atoms are obtained, the distance measures between any two residues can be calculated similarly by taking an average of GDs or EDs from all associated backbone atoms.

\section{Construction of LAD profiles}

LAD is proposed to retain local characteristics of each residue in sequential relationship. The LAD profile for a protein consists of average distance values which are built by employing a sliding window scanning from $\mathrm{N}$ to C-terminus. In this study, we have tried different odd window sizes ranging from 3 to 21 , and the window size of 9 residues provided the best performance on the training dataset (Dataset $L$ from ADiDoS [63]). Hence, a window size of 9 is applied to build all LAD profiles. We have implemented two types of LAD profiles; one is based on ED feature $\left(L A D_{E D}\right.$, Figure $\left.1 d\right)$ and the other is based on $\mathrm{GD}\left(\mathrm{LAD}_{\mathrm{GD}}\right.$, Figure 1e) feature. Given a residue at position $i$ (residue ${ }_{i}$ ) in the sequence, the $\mathrm{LAD}_{i}$ for the residue $_{i}$ is defined by taking average distance from residue $_{i}$ to both side neighbouring residues within the window.

\section{LAD diversity}

The pairwise structure comparison in this study is based on evaluating the similarities of two LAD profiles from two individual proteins. A variation of Smith-Waterman algorithm is performed to obtain the correspondence of residues between two proteins by comparing LADs instead of amino acid contents. The similarity score between two residues, residue $_{i}$ and residue $_{j}$, for dynamic programming is inversely proportional to the absolute difference between $\mathrm{LAD}_{i}$ and $\mathrm{LAD}_{j}$.

The similarity of two proteins is quantified by the result of pairwise profile alignment. A novel scoring function named as LAD diversity $\left(L A D_{\text {div }}\right)$ is proposed, which considers the number of equivalent (aligned) residues $\left(N_{e}\right)$ and the root-mean-square deviation (RMSD) of LADs for aligned residues. The $L A D_{d i v}$ is defined in the following equation where $N_{Q}$ and $N_{S}$ are lengths of the query and the subject proteins respectively. The symbols $D$ and $\alpha$ are used to adjust the effect of RMSD on the $L A D_{d i v}$. Since $N_{e}$ must be less than or equal to $N_{Q}$ and $N_{S}$, the value of $L A D_{\text {div }}$ is between 0 and 1 , and smaller values represent higher similarities.

$$
L A D_{d i v}=1-\frac{N_{e}}{\operatorname{mean}\left(N_{Q}, N_{S}\right)\left[1+\left(\frac{\mathrm{RMSD}}{D}\right)^{\alpha}\right]}
$$

Profile alignment of a similar structure pair tends to hold a low RMSD and a large $N_{e}$, and therefore results in a low $L A D_{d i v}$. For example, a domain swapping protein pair illustrated in the section of self-connection problem possessing (RMSD, $\left.L A D_{\text {div }}\right)$ of $(0.173,0.0004)$ and $(0.454$, 0.02) for $L A D_{E D}$ and $L A D_{G D}$ respectively. Conversely, a dissimilar structure pair possesses a high $L A D_{\text {div }}$ with a large RMSD and a low $N_{e}$ simultaneously. Figure 2 shows an instance of profile alignment for a non-homologous protein pair which possesses different conformations, and accordingly, the $\mathrm{LAD}_{\mathrm{ED}}$ profiles obtained high values of $\left(\mathrm{RMSD}, L A D_{\text {div }}\right)$ as $(1.601,0.955)$ compared to the previous example.

Variables $D$ and $\alpha$ were trained by the Dataset $L$ [63] which contains 706 known domain swapping homologous pairs $(L d s), 487$ common homologous pairs (Lch) and 640 non-homologous pairs $(\operatorname{Lnh})$ of protein structures. Both $L d s$ and $L c h$ were considered as a positive dataset in which each pair was anticipated possessing low $L A D_{\text {div }}$ values. Conversely, $L n h$ was considered as a negative dataset which was expected possessing high $L A D_{\text {div }}$ values for each pairs. Let $L d s_{<0.5}$ and $L c h_{<0.5}$ denote the number of pairs whose $L A D_{d i v}$ is less than 0.5 for both $L d s$ and $L c h$. The $L n h_{\geq 0.5}$ represents the number of pairs whose $L A D_{d i v}$ is larger than or equal to 0.5 . We have evaluated $D$ ranging from 0.1 to 20 with an interval of 0.1 , and a range of 1 to 5 with an interval of 0.5 for $\alpha$. Hence, a total of 1800 $(200 \times 9)$ combinations of $D$ and $\alpha$ were evaluated and the one with maximum $L d s_{<0.5}+L c h_{<0.5}+L n h_{\geq 0.5}$ was selected. Finally, $(D, \alpha)=(1,4.5)$ and $(D, \alpha)=(1.1,5)$ were selected for $L A D_{E D}$ and $L A D_{G D}$ respectively. 


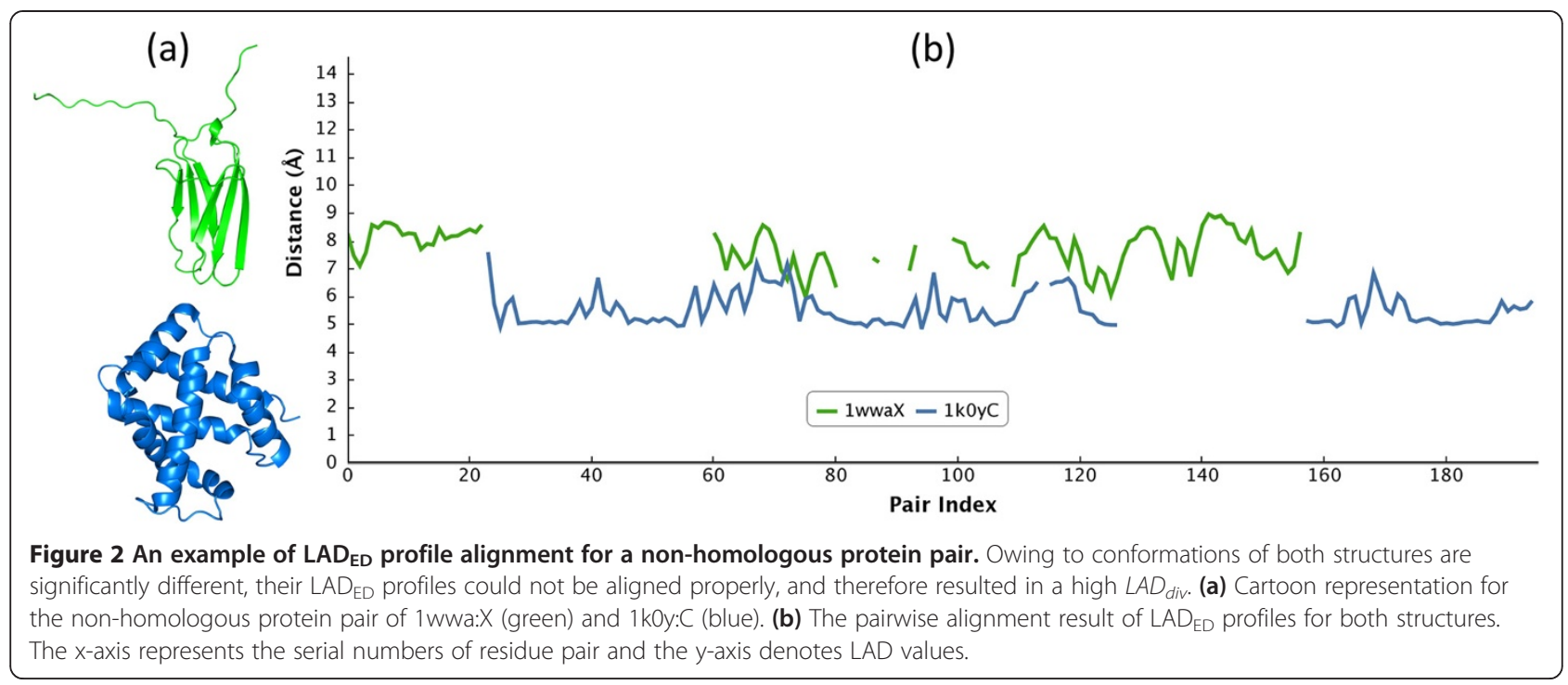

\section{Structural diversity}

There are many different ways to measure protein structural similarity of aligned results, and many of them have been reviewed in [1]. According to our previous research [63], the structure diversity (Struct ${ }_{d i v}$ ) [64] showed superior performances on distinguishing homologous proteins from non-homologous ones upon various structural comparison methods. Therefore, Struct $t_{d i v}$ was employed in this study to compare existing rigid/flexible structural alignment tools with our proposed method. Struct $t_{d i v}$ is defined as:

$$
\text { Struct }_{d i v}=\frac{\text { RMSD }}{\left(\frac{N_{e}}{\operatorname{mean}\left(N_{Q}, N_{S}\right)}\right)^{1.5}}
$$

where RMSD is the root mean square deviation of the distances between the aligned $C \alpha$ atoms. Like $L A D_{d i v}$, structural alignment of a similar structure pair tends to have both low RMSD and large $N_{e}$, and low Struct ${ }_{d i v}$.

\section{Testing datasets}

There were two testing datasets applied in this research to validate our method and compare with existing methods. The first one is Hinge Atlas dataset which contains 2791 protein structures of 214 non-redundant morphs exhibiting hinge bending motions. The lengths of proteins range from 28 to 994 residues. A morph is a group of structures (9 to 32) comprising two homologous proteins with different conformations and several interpolated structures between these two initial structures. About $97 \%$ of morphs in the dataset possess three or less hinge points. Figure 3 shows an example of morph with a large conformational change for the protein GroEL containing 524 residues. Neither $L A D_{E D}$ and $L A D_{G D}$ descriptors are sensitive to the deformation, especially for $\mathrm{LAD}_{\mathrm{ED}}$. The second dataset provided by Liu et al. was a subset of Hinge Atlas [37] and Hinge Atlas Gold datasets, and which was applied in the previous study [36]. The Liu's dataset contains 382 protein structures of 27 groups with large degrees of conformational changes.

\section{Results}

\section{Comparison with structural alignment methods}

LAD descriptors were compared with 2 rigid and 5 flexible structural alignment methods on the Hinge Atlas dataset in terms of retrieving similar structures which belong to the same group (morph) as the query structure. The first structure in each group was regarded as the representative for that group, and the remaining 2577 proteins were considered as query structures. Each query protein compared with 214 representatives, and there were a total of 551478 (2577 x 214) pairwise comparisons. The results for each query were sorted according to the diversity scores $\left(L A D_{d i v}\right.$ or Struct $\left._{d i v}\right)$, and it was regarded as a successful retrieval if the representative belonging to the same group as query proteins was ranked at the first place. The retrieval performance for LAD and other structural alignment methods on the Hinge Atlas dataset were summarized in Table 1. The results have shown that $L A D_{E D}$ and $L A D_{G D}$ performed better than other methods and achieved retrieval success rates of $97.1 \%$ and $95 \%$ respectively. The structural alignment methods generated unsatisfied alignment results even though the relevant structures were successfully retrieved at the first place. For example, all methods ranked the relevant structure of $\mathrm{ff} 0$ at the top position for the query structure of ff9 from the morph group of va2eznA$115 \mathrm{bA}$, and it is a domain-swapped dimer of Cyanovirin- $\mathrm{N}$ (Figure $4 \mathrm{a})$. In this case, $\mathrm{LAD}_{\mathrm{ED}}, \mathrm{LAD}_{\mathrm{GD}}$, FlexProt, FlexSnap and jFATCAT (Figure 4b) could align the protein pair 


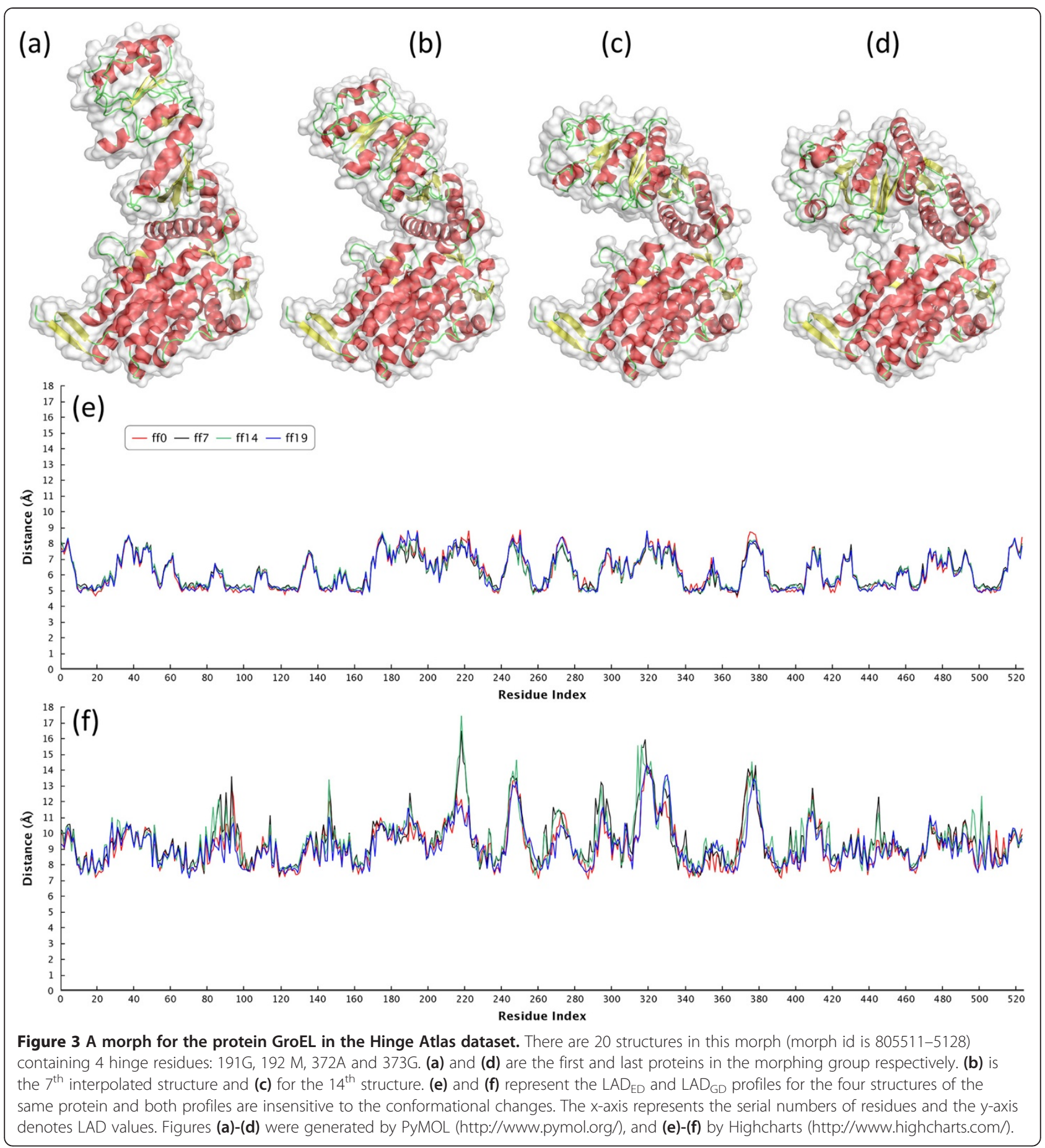

completely, but FASE (Figure 4c), Fast (Figure 4d), Matt-Rigid (Figure 4e) and Matt-Flexible (Figure 4f) only aligned half portion of the structure.

In addition to the measure of successful retrieval rates, we also evaluated the performances for the Hinge Atlas dataset based on the precision-recall curve of 11-point interpolated average precision which is a common measurement in information retrieval systems [65]. It should be noted that the 214 representatives were treated as query structures individually, and each of them compared with the remaining 2577 structures in order to search structures belonging to the same group. A precision rate is the fraction of retrieved structures that are relevant to the query protein, and a recall rate is the fraction of relevant structures that are successfully 
Table 1 Retrieval performances of 2577 queries for different methods on the Hinge Atlas dataset

\begin{tabular}{lcc}
\hline Method & Number of successful retrieval & Success rate (\%) \\
\hline LAD $_{E D}$ & 2502 & 97.1 \\
LAD $_{G D}$ & 2447 & 95.0 \\
Matt-Flexible & 2342 & 90.9 \\
FlexSnap & 2329 & 90.4 \\
FASE & 2282 & 88.6 \\
jFATCAT & 2241 & 87.0 \\
FAST $^{*}$ & 2234 & 86.7 \\
Matt-Rigid $^{*}$ & 2185 & 84.8 \\
FlexProt $^{*}$ & 2167 & 84.1
\end{tabular}

"Rigid alignment method.

The results are ordered by the success rates and show that both $L A D_{E D}$ and $L A D_{G D}$ outperform other methods.

retrieved. Precision and recall rates are defined in the following equations:

$$
\begin{aligned}
& \text { Precision }=\frac{T P}{T P+F P} \\
& \text { Recall }=\frac{T P}{T P+F N}
\end{aligned}
$$

True positive $(T P)$ is the number of successful retrieved structures; false positive $(F P)$ represents the number of inaccurately retrieved structures; false negative $(F N)$ denotes the number of structures belonging to the same group as query but not being retrieved. The interpolated precision for a specific recall $r$ is defined as the maximum precision over any recall $r^{\prime} \geq r$ [65]. For each query, a set of 11 interpolated precisions at 11 recall levels $(0,0.1,0.2 \ldots 1)$ were determined, then averages of interpolated precisions for 214 queries at each level were calculated. According to the precision-recall curves (see Figure 5), both $\mathrm{LAD}_{\mathrm{ED}}$ and $\mathrm{LAD}_{\mathrm{GD}}$ outperformed other methods since they possessed larger area under the curve.

$R$-Precision and Mean Average Precision (MAP) are the other common quantitative measures for evaluating overall performance of information retrieval systems. If there are total $R$ relevant structures for a query, $R$-Precision is defined as the number of relevant structures in the top $R$ retrieved structures divided by $R$. For a query, Average Precision is an average of precisions for each relevant structure. MAP is defined as the mean of the Average Precisions for a set of queries. For more details of calculating these measures please refer to [65]. The

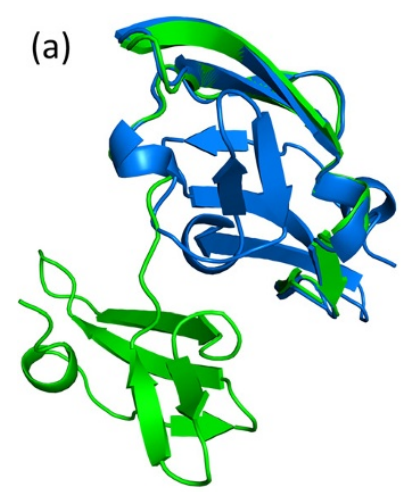

(d)

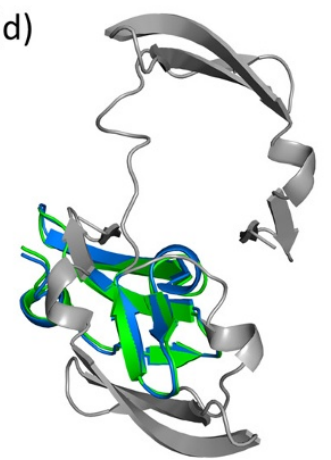

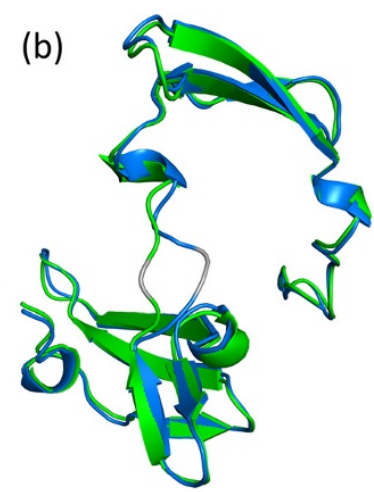

(b)

(e)

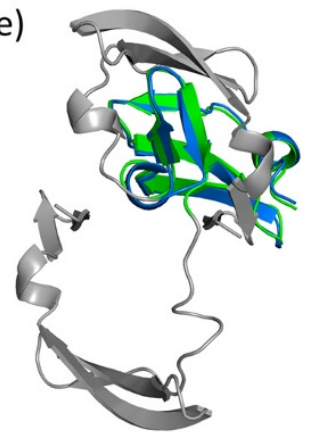

(c)

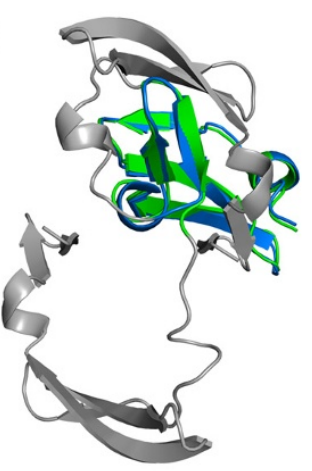

(f)

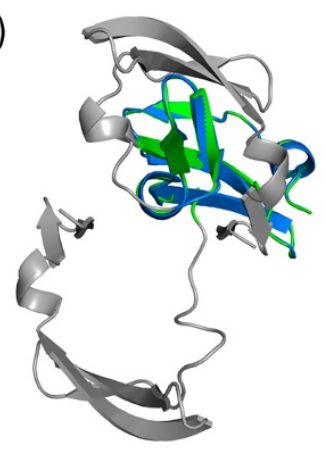

Figure 4 An example of successful retrieval but with poor structure alignments. The structure pair is from the morphing group of va2eznA-115bA in the Hinge Atlas dataset. (a) The open-form (green, ffg) and closed-form (blue, ffo) of Cyanovirin-N. (b) to (f) are structure alignments generated by jFATCAT, FASE, Fast, Matt-Rigid and Matt-Flexible respectively. The non-aligned regions are colored by gray. All methods ranked the closed-form of Cyanovirin- $\mathrm{N}$ at the top of 214 representative structures when the open-form of Cyanovirin-N as a query; nevertheless, FASE, Fast, Matt-Rigid and Matt-Flexible only aligned half portion of the query protein. 


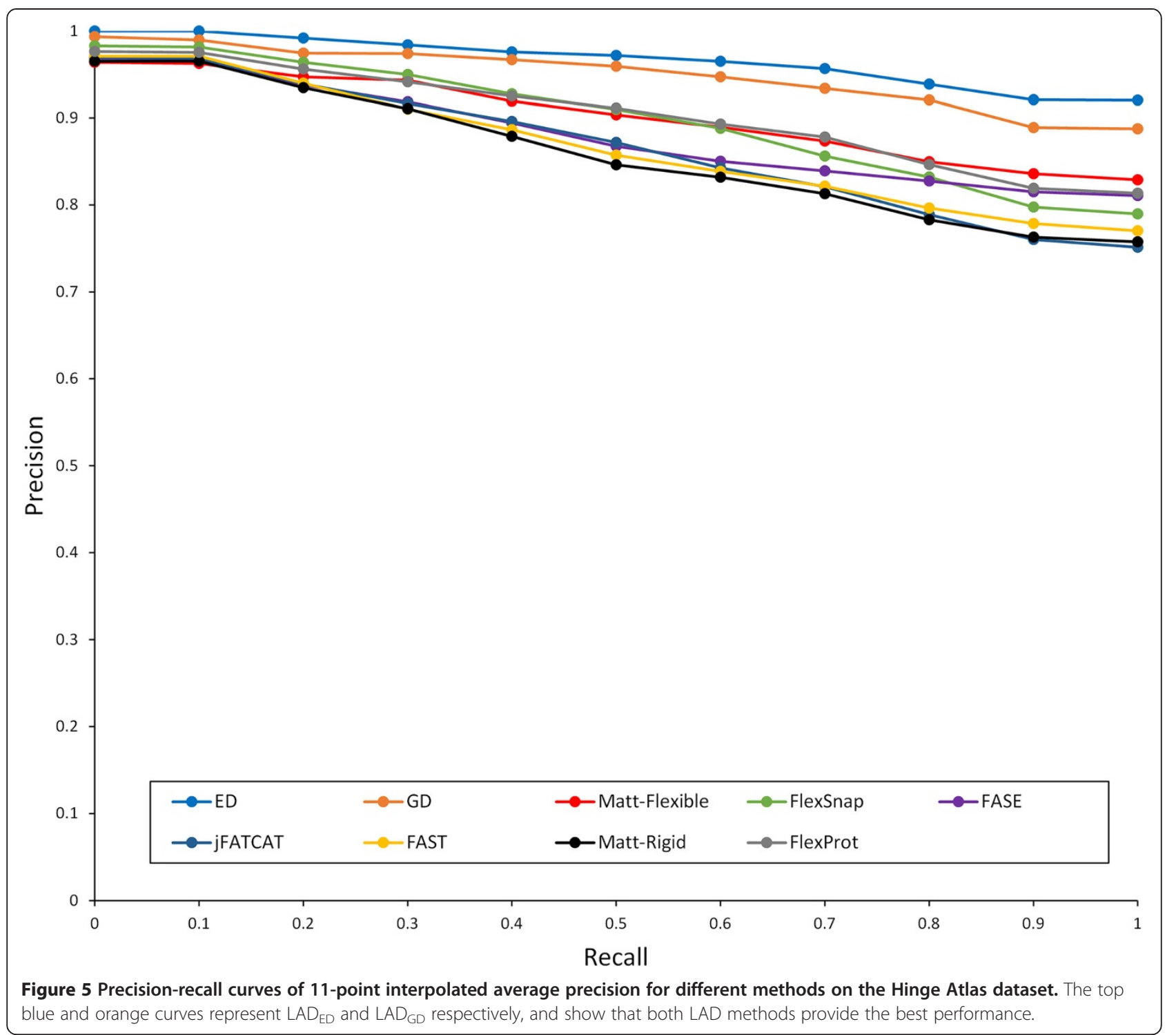

Table 2 Retrieval performances of $\mathbf{2 1 4}$ queries for different methods on the Hinge Atlas dataset

\begin{tabular}{|c|c|c|}
\hline Method & Average $R$-precision (\%) & Mean average precision (\%) \\
\hline$\angle A D_{E D}$ & 95.54 & 96.67 \\
\hline$L A D_{G D}$ & 93.53 & 94.95 \\
\hline Matt-Flexible & 87.55 & 89.62 \\
\hline FlexSnap & 86.97 & 89.71 \\
\hline FASE & 84.97 & 87.40 \\
\hline jFATCAT & 83.36 & 86.23 \\
\hline FAST & 82.81 & 86.16 \\
\hline Matt-Rigid & 82.24 & 85.33 \\
\hline FlexProt & 87.14 & 89.98 \\
\hline
\end{tabular}

average $R$-Precision and MAP of 214 queries for different methods are shown in Table 2. The results have shown that both $\mathrm{LAD}_{\mathrm{ED}}$ and $\mathrm{LAD}_{\mathrm{GD}}$ performed superior to other methods, and $\mathrm{LAD}_{\mathrm{ED}}$ achieves an average of 95.54\% for $R$-Precision and $96.67 \%$ for MAP.

\section{Comparison with non-alignment methods}

The Liu's dataset was employed to compare LAD descriptor with non-alignment methods. In order to compare with the results in [36], only the top 64 retrieved structures for each query were used to compute the precision and recall rates. The $\mathrm{F}_{1}$-measure is the harmonic mean of recall and precision rates defined as:

$$
F_{1}-\text { measure }=\frac{2 \times \text { Precision } \times \text { Recall }}{\text { Precision }+ \text { Recall }}
$$


where the maximum value is 1 . In contrast to the arithmetic mean, both precision and recall rates need to be high to obtain a high $\mathrm{F}_{1}$-measure. The retrieval performance of $F_{1}$-measure is listed in Table 3. $\mathrm{LAD}_{\mathrm{ED}}$ and $\mathrm{LAD}_{\mathrm{GD}}$ achieved $43.27 \%$ and $43.18 \%$ of $\mathrm{F}_{1}$-measure respectively and outperformed the other 7 non-alignment methods with a highest $\mathrm{F}_{1}$-measure of $37.04 \%$.

\section{Discussion}

\section{Self-connection problem}

Figure 6 is an example of bona fide domain swapping protein pair holding self-connection on surface caused by a large hinge bending motion. The difficulty is that a selfconnection leads to topology changes, hence the inner distance method considering all landmark points cannot solve this problem $[35,36]$. However, this type of deformation can be overcome by our proposed descriptor especially for $\mathrm{LAD}_{\mathrm{ED}}$ approach since an LAD only considers the local geometric properties which are not sensitive to global topology changes. Figure $6 \mathrm{~d}$ and Figure $6 \mathrm{e}$ have shown a high consistency of $\mathrm{LAD}_{\mathrm{ED}}$ and $\mathrm{LAD}_{\mathrm{GD}}$ profiles between openform (PDB code: 1a2w, chain A) and close-form (PDB code: 5 rsa, chain A) of Ribonuclease A respectively. It is obvious that $L A D_{E D}$ is more consistent than $L A D_{G D}$ in this case, but both $L A D_{\text {div }}$ are close to zero representing highly similar conformations. The (RMSD, $L A D_{\text {div }}$ ) for $L A D_{E D}$ is $(0.173,0.0004)$ and $(0.454,0.02)$ for $\mathrm{LAD}_{\mathrm{GD}}$.

In general, LAD descriptors are insensitive to selfconnection cases; however, an $\mathrm{LAD}_{\mathrm{GD}}$ profile is sometimes not consistent at the location of self-connecting regions. Given another domain swapping example in Figure 6, an open-form Ribonuclease A (PDB code: 1js0, chain A) changes to a closed-form (PDB code: 3di8, chain A). The swapped domain (yellow surface) bends and intertwines with the protein body (blue surface) via conformational changes of highly flexible hinge loops (red surface) (see Figure 7a and Figure $7 b$ ). In Figure $7 c$, it is obvious that the $\mathrm{LAD}_{\mathrm{ED}}$ varies slightly between the open- and close-form states from H105

Table 3 Comparison with non-alignment methods on Liu's dataset

\begin{tabular}{lc}
\hline Method & $\mathbf{F}_{\mathbf{1}}$-measure (\%) \\
\hline $\mathrm{LAD}_{\mathrm{ED}}$ & 43.27 \\
$\mathrm{LAD}_{\mathrm{GD}}$ & 43.18 \\
Diffusion distance (DD) & 37.04 \\
Inner distance (ID) & 35.83 \\
Shape distribution (SD) & 28.40 \\
Euclidean distance (ED) & 28.81 \\
Solid angle histogram (SAH) & 25.69 \\
Geodesic distance (GD) & 26.42 \\
Spherical harmonic descriptor (SHD) & 23.93 \\
\hline
\end{tabular}

The results are taken from [36] except $L A D_{E D}$ and $L A D_{G D}$. to A109 residues (magenta rectangle). In contrast, the $\mathrm{LAD}_{\mathrm{GD}}$ of close-form state is higher than that of open-form state at corresponding highlighted regions (see Figure $7 d$ ). For a detailed illustration, it can be imagined a path from the residue $\mathrm{H} 105$ to its +3 position (V108). When the swapped domain locates apart from the protein body in the open-form state, the GD between these two residues is the shortest path along the white surface. The GD and ED between the two residues in the open-form state are $11.12 \AA$ and $10.37 \AA$ respectively. However, the path was changed while the swapped domain bending to the body and intertwining with the white surface region forming a selfconnection case. The GD is increased significantly due to an additional mountain (yellow region in Figure 7b) obstructing the original path from residue H105 to V108. The ED maintained high similarity since its path directly passed through the mountain instead of along on the surface. The GD and ED between the two residues of the close-form state are $16.77 \AA$ and 9.57 respectively. This phenomenon is the main reason why an $\mathrm{LAD}_{\mathrm{GD}}$ descriptor more sensitive to the topological changes than $\mathrm{LAD}_{\mathrm{ED}}$.

\section{Differences between the previous and proposed ED/GD based methods}

In previous studies [34-36], ED and GD were shown to be sensitive to shape deformation and not feasible for flexible molecular shape comparison. However, it is interesting that relying on the proposed LAD methods, both features become insensitive to topological changes and reveal deformation invariant properties to tackle with the flexibility problems. The reason for sensitive ED and GD features in previous studies is that both distances were computed among all global landmark points. On the contrary, the LAD exploits the characterization of local geometric features for each residue and its neighbouring residues. Therefore, ED and GD features become much less sensitive to global topological changes.

\section{Computational time}

Pairwise comparison of LAD profiles was performed by a modification of Smith-Waterman algorithm and possessed the same time complexity. The goal of a sequence alignment problem is to identify the correspondence of residues between two given proteins, while a structure alignment emphasizes on finding both an alignment and a spatial superposition. Possible combinations of corresponding residues are countable while possibilities of special superposition are innumerable. Therefore, the computational complexity of the proposed algorithm is inherently less than most commonly used structure alignment methods [66]. The LAD algorithm was implemented by $\mathrm{C \#}$.NET running on an Intel Core i5-2500 $3.3 \mathrm{GHz}$ computer with $16 \mathrm{~GB}$ ram. According to the 551478 pairwise comparisons mentioned in the result 


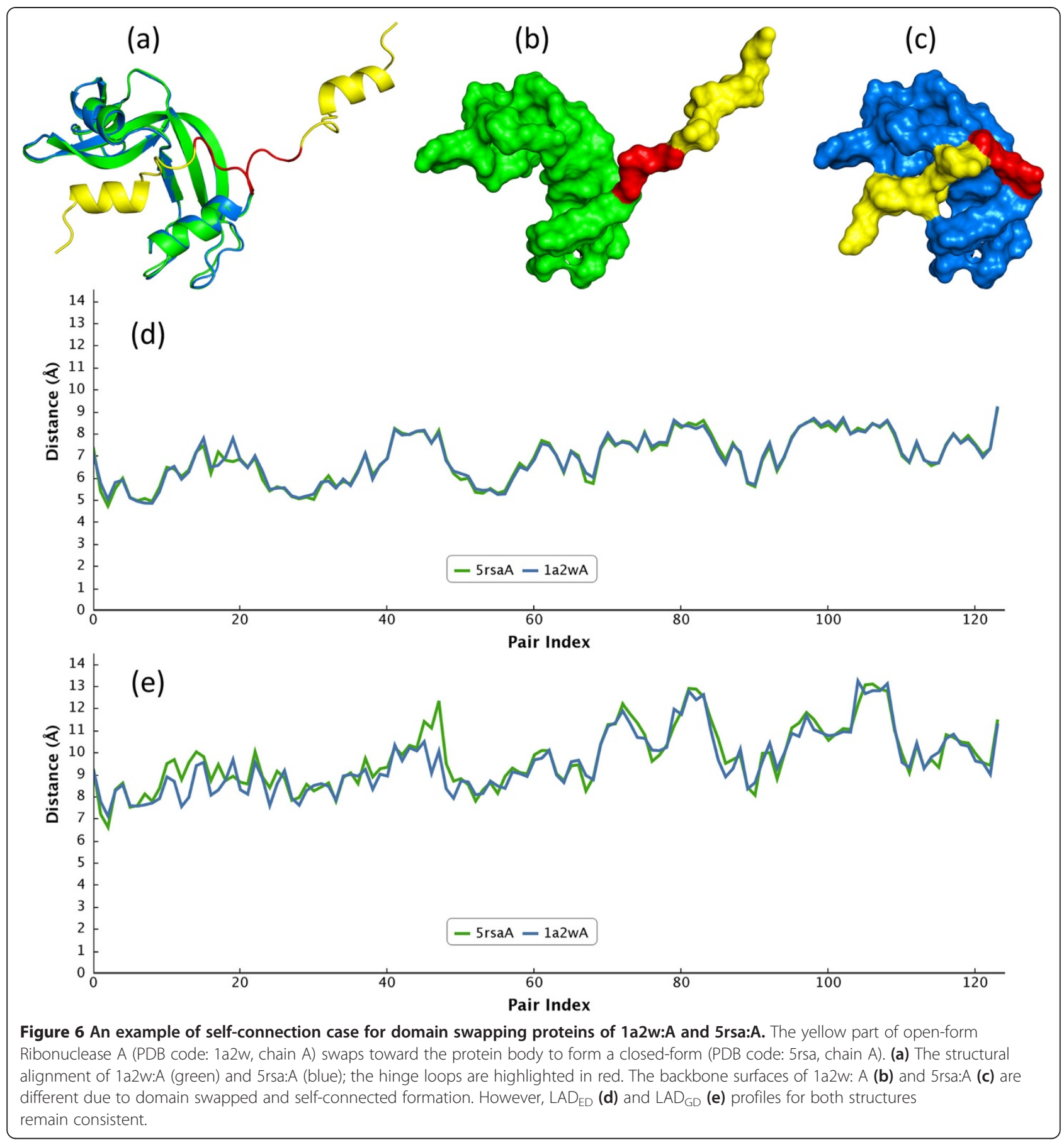

section, it only cost an average computational time of 3.896 and 4.828 milliseconds per comparison for $L A D_{E D}$ and $\mathrm{LAD}_{\mathrm{GD}}$ profiles respectively.

\section{Conclusions}

We proposed a novel profile-based alignment method, named LAD, for pairwise flexible protein structure comparison. It can be constructed in a sense of any kind of spatial measures of local neighbouring residues within a specific sliding window. Here, GD and ED were used to build $\mathrm{LAD}_{\mathrm{GD}}$ and $\mathrm{LAD}_{\mathrm{ED}}$ profiles. The idea of LAD improves the ED- and GD-based descriptors which were previously shown to be sensitive to molecular shape deformation, in particular to topologically structural changes. The effectiveness of LAD descriptor has been evaluated on two datasets of hinge bending motions from the MolMovDB. Our methods are robust to deformed flexible molecules and achieve good performance regarding assignment 
(a)

(c)

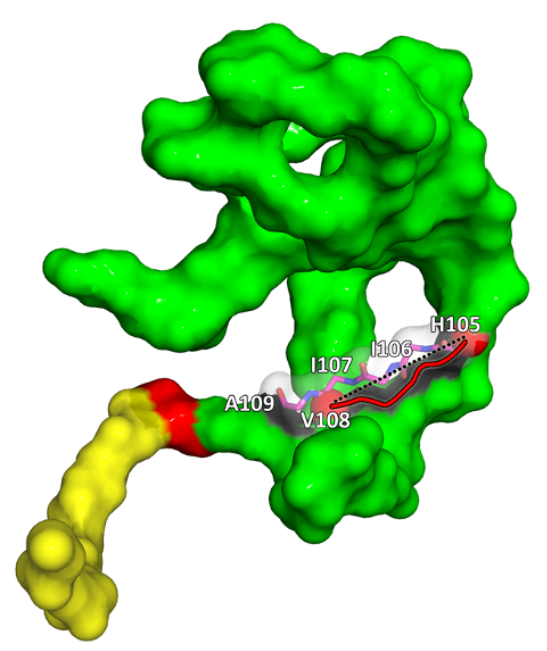

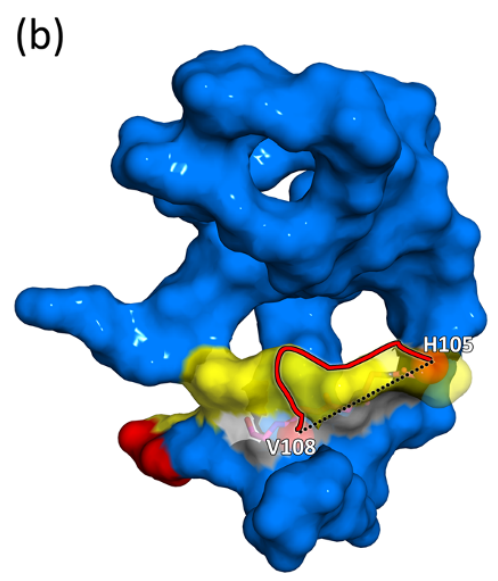

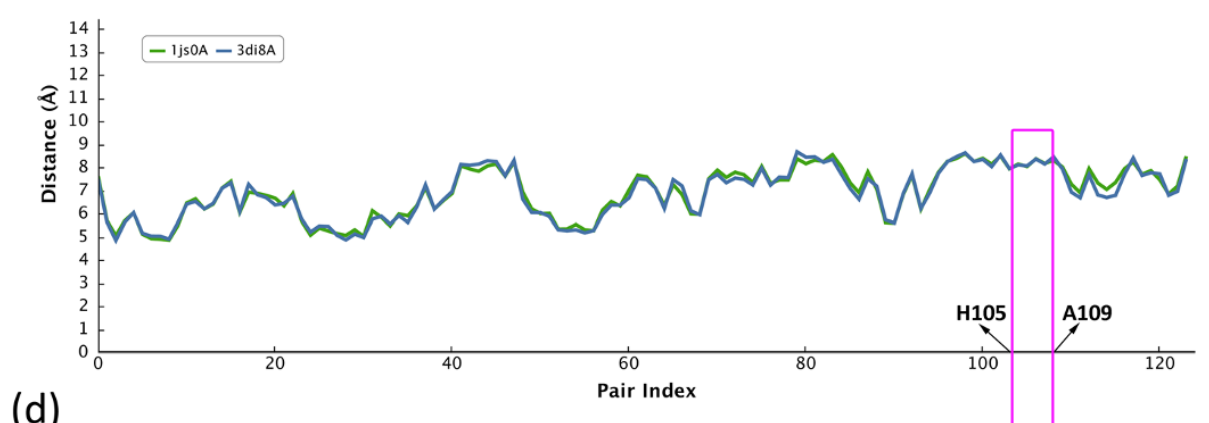

(d)

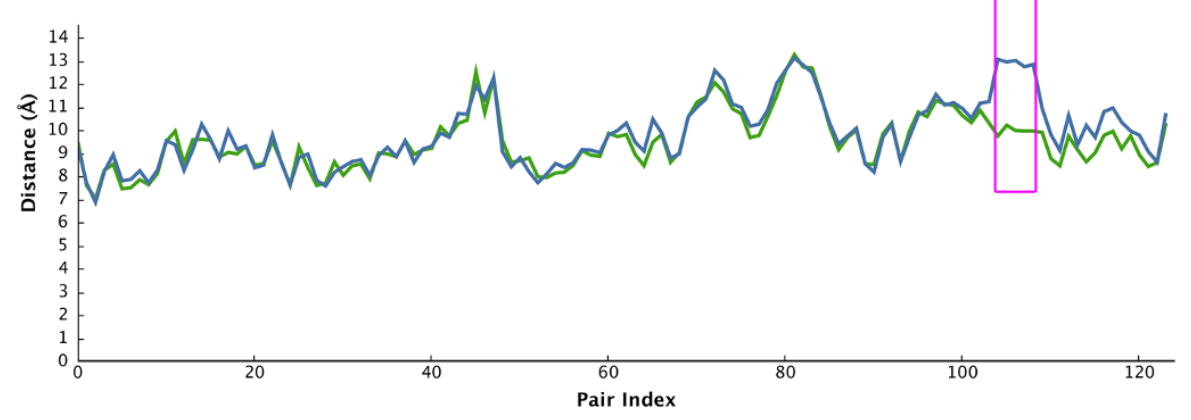

Figure 7 Illustrating the variation between $L A D_{E D}$ and $L A D_{G D}$ for a self-connection case.The 3D domain-swapped Ribonuclease $A$ consists of a protein body (green/blue surface), a hinge loop (red surface) and a swapped domain (yellow surface). (a) The open-form Ribonuclease $A$ (PDB code: 1js0, chain A) (b) The domain-swapped closed homolog of (a) (PDB code: 3di8, chain A). (c) and (d) are LAD $D_{E D}$ and $L A D_{G D}$ profiles of both closed- and open-form structures respectively. The red solid curve of (a) and (b) denotes a GD path, which is the shortest path along the surface (white surface region) connecting two residues H105 and V108 (red spheres). The residues from H105 to A109 of both proteins are shown as magenta sticks and highlighted within a magenta box in (c) and (d). The black dashed line of (a) and (b) indicates the ED path between the residues $\mathrm{H} 105$ and $\mathrm{V} 108$. Note that the magenta box has shown that the $L A D_{G D}$ profile is more sensitive at the topological changed locations than the LADED profile.

of the queries to different classes of molecules with conformational changes, and the results have shown superior performance compared to existing alignment- and nonalignment-based tools. Finally, the reasons of LAD descriptor being insensitive to flexible proteins with selfconnection circumstance was described by taking 3D domain swapping cases as examples, and further discussion of $L A D_{E D}$ possessing more robust properties than $L A D_{G D}$ was also explained. Required computational time for pairwise $\mathrm{LAD}_{\mathrm{ED}} / \mathrm{LAD}_{\mathrm{GD}}$ profile comparisons was analyzed to demonstrate its feasibility for constructing an on-line structure comparison system. The proposed descriptor is indeed effective in retrieving deformed proteins and it could be an alternative approach for database search, discovery of previously unknown conformational relationships, and reorganization of protein structure classification. 


\section{Availability of supporting data}

The training and testing datasets for our method can be obtained from previously published papers by $\mathrm{Chu} \mathrm{CH}$ [63] and Flores SC [37,38].

\section{Abbreviations}

LAD: Local average distance; ED: Euclidean distance; GD: Geodesic distance; MAP: Mean average precision.

\section{Competing interests}

The authors declare that they have no competing interests.

\section{Authors' contributions}

HWW, CHC and TWP conceived the algorithm. HWW and $\mathrm{CHC}$ implemented the algorithm, performed the experiments and wrote the manuscript. TWP and WCW proofread and revised the manuscript. All authors read and approved the final manuscript.

\section{Acknowledgements}

Thanks to Mr. Yueh-Lin Tsai for initiating the research topic and thanks to Professor Liu for providing the testing dataset of Hinge Atlas. This work was supported by the Center of Excellence for the Oceans from National Taiwan Ocean University and the National Science Council, Taiwan (NSC101-2627-B-019-003 and NSC1022321-B-019-001 to Tun-Wen Pai, NSC102-2325-B-007-001 to Wen-Ching Wang)

\section{Author details}

${ }^{1}$ Department of Computer Science and Engineering, National Taiwan Ocean University, Keelung, Taiwan. ${ }^{2}$ Biomedical Science and Engineering Center, National Tsing Hua University, Hsinchu, Taiwan. ${ }^{3}$ Institute of Molecular and Cellular Biology and Department of Life Science, National Tsing Hua University, Hsinchu, Taiwan.

Received: 21 September 2013 Accepted: 22 March 2014 Published: 2 April 2014

\section{References}

1. Hasegawa H, Holm L: Advances and pitfalls of protein structural alignment. Curr Opin Struct Biol 2009, 19(3):341-348.

2. Shindyalov IN, Bourne PE: Protein structure alignment by incremental combinatorial extension (CE) of the optimal path. Protein Eng 1998 , 11(9):739-747.

3. Holm L, Park J: DaliLite workbench for protein structure comparison Bioinformatics 2000, 16(6):566-567.

4. Wang S, Zheng WM: CLePAPS: fast pair alignment of protein structures based on conformational letters. J Bioinform Comput Biol 2008 6(2):347-366.

5. Zhu J, Weng Z: FAST: a novel protein structure alignment algorithm. Proteins 2005, 58(3):618-627.

6. Zhang Y, Skolnick J: TM-align: a protein structure alignment algorithm based on the TM-score. Nucleic Acids Res 2005, 33(7):2302-2309.

7. Gelly JC, Joseph AP, Srinivasan N, de Brevern AG: iPBA: a tool for protein structure comparison using sequence alignment strategies. Nucleic Acids Res 2011, 39(Web Server issue):W18-23.

8. Shah SB, Sahinidis NV: SAS-Pro: simultaneous residue assignment and structure superposition for protein structure alignment. PloS one 2012, 7(5):e37493.

9. Guda C, Lu S, Scheeff ED, Bourne PE, Shindyalov IN: CE-MC: a multiple protein structure alignment server. Nucleic Acids Res 2004, 32(Web Server issue):W100-103

10. Konagurthu AS, Whisstock JC, Stuckey PJ, Lesk AM: MUSTANG: a multiple structural alignment algorithm. Proteins 2006, 64(3):559-574.

11. Lupyan D, Leo-Macias A, Ortiz AR: A new progressive-iterative algorithm for multiple structure alignment. Bioinformatics 2005, 21(15):3255-3263.

12. Wang S, Peng J, Xu J: Alignment of distantly related protein structures: algorithm, bound and implications to homology modeling. Bioinformatics 2011, 27(18):2537-2545.

13. Liu X, Zhao YP, Zheng WM: CLEMAPS: multiple alignment of protein structures based on conformational letters. Proteins 2008, 71(2):728-736.

14. Shatsky M, Nussinov R, Wolfson HJ: A method for simultaneous alignment of multiple protein structures. Proteins 2004, 56(1):143-156.
15. Ye $Y$, Godzik A: Multiple flexible structure alignment using partial order graphs. Bioinformatics 2005, 21(10):2362-2369.

16. Shealy $\mathrm{P}$, Valafar $\mathrm{H}$ : Multiple structure alignment with msTALI. BMC bioinformatics 2012, 13:105.

17. Rashin AA, Rashin AH, Jernigan RL: Diversity of function-related conformational changes in proteins: coordinate uncertainty, fragment rigidity, and stability. Biochemistry 2010, 49(27):5683-5704.

18. Nigham A, Hsu D: Protein conformational flexibility analysis with noisy data. J Comput Biol 2008, 15(7):813-828.

19. Shatsky M, Nussinov R, Wolfson HJ: Flexible protein alignment and hinge detection. Proteins 2002, 48(2):242-256.

20. Shatsky M, Nussinov R, Wolfson HJ: FlexProt: alignment of flexible protein structures without a predefinition of hinge regions. J Comput Biol 2004, 11(1):83-106.

21. Ye $Y$, Godzik A: Flexible structure alignment by chaining aligned fragment pairs allowing twists. Bioinformatics 2003, 19(2):ii246-255.

22. Veeramalai M, Ye Y, Godzik A: TOPS++FATCAT: fast flexible structural alignment using constraints derived from TOPS + strings model. BMC bioinformatics 2008, 9:358.

23. Vesterstrom J, Taylor WR: Flexible secondary structure based protein structure comparison applied to the detection of circular permutation. J Comput Biol 2006, 13(1):43-63.

24. Salem S, Zaki MJ, Bystroff C: FlexSnap: flexible non-sequential protein structure alignment. Algorithms for molecular biology: AMB 2010, 5:12.

25. Menke M, Berger B, Cowen L: Matt: local flexibility aids protein multiple structure alignment. PLOS Comput Biol 2008, 4(1):e10

26. Berbalk C, Schwaiger CS, Lackner P: Accuracy analysis of multiple structure alignments. Protein Sci 2009, 18(10):2027-2035.

27. Sael L, Li B, La D, Fang Y, Ramani K, Rustamov R, Kihara D: Fast protein tertiary structure retrieval based on global surface shape similarity. Proteins 2008, 72(4):1259-1273.

28. Liu YS, Wang M, Paul JC, Ramani K: 3DMolNavi: a web-based retrieval and navigation tool for flexible molecular shape comparison. BMC bioinformatics 2012, 13:95.

29. Venkatraman $V$, Sael L, Kihara D: Potential for protein surface shape analysis using spherical harmonics and 3D Zernike descriptors. Cell Biochem Biophys 2009, 54(1-3):23-32.

30. Daras P, Zarpalas D, Axenopoulos A, Tzovaras D, Strintzis MG: Threedimensional shape-structure comparison method for protein classification. IEEE/ACM Trans Comput Biol Bioinform 2006, 3(3):193-207.

31. Sael $L$, Kihara D: Improved protein surface comparison and application to low-resolution protein structure data. BMC bioinformatics 2010, 11(11):S2

32. Abu Deeb Z, Adjeroh DA, Jiang BH: Protein surface characterization using an invariant descriptor. International journal of biomedical imaging 2011, 2011:918978.

33. Yin S, Proctor EA, Lugovskoy AA, Dokholyan NV: Fast screening of protein surfaces using geometric invariant fingerprints. Proc Natl Acad Sci USA 2009, 106(39):16622-16626

34. Fang Y, Liu YS, Ramani K. Three dimensional shape comparison of flexible proteins using the local-diameter descriptor. BMC Struct Bio/ 2009, 9:29.

35. Liu YS, Fang Y, Ramani K: IDSS: deformation invariant signatures for molecular shape comparison. BMC bioinformatics 2009, 10:157.

36. Liu YS, Li Q, Zheng GQ, Ramani K, Benjamin W: Using diffusion distances for flexible molecular shape comparison. BMC bioinformatics 2010, 11:480.

37. Flores SC, Lu L, Yang J, Carriero N, Gerstein MB: Hinge Atlas: relating protein sequence to sites of structural flexibility. BMC bioinformatics 2007, 8:167

38. Flores S, Echols N, Milburn D, Hespenheide B, Keating K, Lu J, Wells S, Yu EZ Thorpe M, Gerstein M: The database of macromolecular motions: new features added at the decade mark. Nucleic Acids Res 2006, 34(Database issue):D296-301.

39. Lee B, Richards FM: The interpretation of protein structures: estimation of static accessibility. J Mol Biol 1971, 55(3):379-400.

40. Connolly ML: Analytical molecular surface calculation. J App/ Crystallogr 1983, 16(5):548-558.

41. Richards FM: Areas, volumes, packing and protein structure. Annu Rev Biophys Bioeng 1977, 6:151-176.

42. Connolly ML: Molecular surfaces: a review, Network science 1996. http://www. netsci.org/Science/Compchem/feature14.html (accessed 18 Feb. 2014).

43. Tsodikov OV, Record MT Jr, Sergeev W: Novel computer program for fast exact calculation of accessible and molecular surface areas and average surface curvature. J Comput Chem 2002, 23(6):600-609. 
44. Can T, Chen Cl, Wang YF: Efficient molecular surface generation using level-set methods. J Mol Graph Model 2006, 25(4):442-454.

45. Liang J, Edelsbrunner H, Fu P, Sudhakar PV, Subramaniam S: Analytical shape computation of macromolecules: I: molecular area and volume through alpha shape. Proteins 1998, 33(1):1-17.

46. Albou LP, Schwarz B, Poch O, Wurtz JM, Moras D: Defining and characterizing protein surface using alpha shapes. Proteins 2009, 76(1):1-12.

47. Ryu J, Park R, Kim D-S: Molecular surfaces on proteins via beta shapes. Comput Aided Des 2007, 39(12):1042-1057.

48. Xu D, Zhang Y: Generating triangulated macromolecular surfaces by Euclidean Distance Transform. PloS one 2009, 4(12):e8140.

49. Decherchi S, Rocchia W: A general and robust ray-casting-based algorithm for triangulating surfaces at the nanoscale. PloS one 2013, 8(4):e59744.

50. Yu Z, Holst MJ, Cheng Y, McCammon JA: Feature-preserving adaptive mesh generation for molecular shape modeling and simulation. J Mol Graph Model 2008, 26(8):1370-1380.

51. Connolly ML: The molecular surface package. J Mol Graph 1993, 11(2):139-141.

52. Rocchia W, Sridharan S, Nicholls A, Alexov E, Chiabrera A, Honig B: Rapid grid-based construction of the molecular surface and the use of induced surface charge to calculate reaction field energies: applications to the molecular systems and geometric objects. J Comput Chem 2002, 23(1):128-137.

53. Jordan RA, El-Manzalawy Y, Dobbs D, Honavar V: Predicting protein-protein interface residues using local surface structural similarity. BMC bioinformatics 2012, 13:41

54. Ma B, Elkayam T, Wolfson H, Nussinov R: Protein-protein interactions: structurally conserved residues distinguish between binding sites and exposed protein surfaces. Proc Natl Acad Sci U S A 2003, 100(10):5772-5777.

55. Yan C, Honavar V, Dobbs D: Identification of interface residues in protease-inhibitor and antigen-antibody complexes: a support vector machine approach. Neural computing \& applications 2004, 13(2):123-129.

56. Singh $\mathrm{H}$, Ahmad S: Context dependent reference states of solvent accessibility derived from native protein structures and assessed by predictability analysis. BMC Struct Biol 2009, 9:25.

57. Tien MZ, Meyer AG, Sydykova DK, Spielman SJ, Wilke CO: Maximum allowed solvent accessibilites of residues in proteins. PloS one 2013, 8(11):e80635.

58. Chakravarty S, Varadarajan R: Residue depth: a novel parameter for the analysis of protein structure and stability. Structure 1999, 7(7):723-732.

59. Pintar $A$, Carugo O, Pongor S: Atom depth as a descriptor of the protein interior. Biophys J 2003, 84(4):2553-2561.

60. Pintar A, Carugo O, Pongor S: Atom depth in protein structure and function. Trends Biochem Sci 2003, 28(11):593-597.

61. Sanner MF, Olson AJ, Spehner JC: Reduced surface: an efficient way to compute molecular surfaces. Biopolymers 1996, 38(3):305-320.

62. Garland $M$, Heckbert PS: Surface simplification using quadric error metrics. In ACM SIGGRAPH; 1997:209-216.

63. Chu CH, Lo WC, Wang HW, Hsu YC, Hwang JK, Lyu PC, Pai TW, Tang CY: Detection and alignment of 3D domain swapping proteins using angle-distance image-based secondary structural matching techniques. PloS one 2010, 5(10):e13361.

64. LU G: TOP: a new method for protein structure comparisons and similarity searches. J App/ Crystallogr 2000, 33(1):176-183.

65. Manning $C D$, Raghavan $P$, Schütze H: Introduction to information retrieval. New York, NY USA: Cambridge University Press; 2008.

66. Poleksic A: Optimal pairwise alignment of fixed protein structures in subquadratic time. J Bioinform Comput Biol 2011, 9(3):367-382.

\section{Submit your next manuscript to BioMed Central and take full advantage of:}

- Convenient online submission

- Thorough peer review

- No space constraints or color figure charges

- Immediate publication on acceptance

- Inclusion in PubMed, CAS, Scopus and Google Scholar

- Research which is freely available for redistribution

Submit your manuscript at www.biomedcentral.com/submit
C Biomed Central 